optic neuropathy (LHON). The disease results in sudden loss of vision between adolescence and adulthood, due to death of the optic nerve. Affected relatives are invariably related through the maternal lineage. Using the polymerase chain reaction to facilitate analysis of segments of mtDNA from patients belonging to several different pedigrees, Wallace et al. ${ }^{2}$ could show that each affected individual contains only a single type of mtDNA. Sequence analysis showed that a $G$ to $A$ transition, converting a highly conserved arginine residue to a histidine in the gene for subunit 4 of NADH dehydrogenase, was the only change that could be correlated with the presence of the disease in 33 individuals from 9 pedigrees. Although not yet formally proven, it seems reasonable to assume that this subtle missense mutation affects the efficiency of either electron flow or energy transduction through the enzyme, thus reducing ATP production enough within the optic nerve to cause premature cell death and blindness.

A more complex clinical picture is presented by patients with a maternally inherited mitochondrial encephalomyopathy that results in myoclonic epilepsy associated with ragged red fibres (MERRF). Patients display varying degrees of mitochondrial abnormality, as manifested by impaired muscle oxidative metabolism and reduced respiratory chain activity $^{3}$. Generally speaking, the order of affected tissues is consistent with their reliance on mitochondrial function and the severity of the biochemical defects correlates with the severity of the symptoms. Molecular analysis of mtDNA, although excluding large deletions or insertions, has so far failed to pinpoint the change responsible. The wide range of clinical symptoms suggests, however, that the patients are in fact mosaics of wild type and mutant mtDNAs, the relative proportion of each type of molecule varying along the lineage, so determining the severity of the affliction. The disease is thus most probably caused by one or more deleterious mutations, that can only be maintained in the heteroplasmic state, in association with wild type mtDNA.

Heteroplasmy of mtDNA is a common feature of a number of other mitochondrial abnormalities associated with a wide 100 years ago

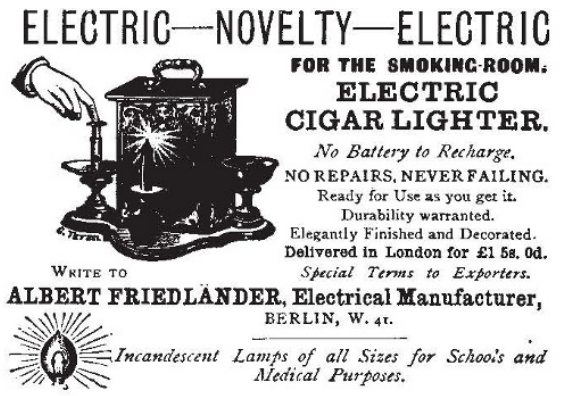

From Nature 40, cci; 17 October 1889.

\title{
DNA amplification
}

\begin{abstract}
"The principles for extensive synthesis of the duplexed tRNA genes which emerge from the present work are the following. The DNA duplex would be denatured to form single strands. This denaturation step would be carried out in the presence of a sufficiently large excess of the two appropriate primers. Upon cooling, one would hope to obtain two structures, each containing the full length of the template strand appropriately complexed with the primer. DNA polymerase will be added to complete the process of repair replication. Two molecules of the original duplex should result. The whole cycle could be repeated, there being added every time a fresh dose of the enzyme. It is however, possible that upon cooling after denaturation of the DNA duplex, renaturation to form the original duplex would predominate over the template-primer complex formation. If this tendency could not be circumvented by adjusting the concentrations of the primers, clearly one would have to resort to the separation of the strands and then carry out repair replication. After every cycle of repair replication, the process of strand separation would have to be repeated. Experiments based on these lines of thought are in progress."
\end{abstract}

Members of the growing band of biomedical scientists who use PCR - the "polymerase chain reaction' for amplifying specific DNA sequences, described by Kary Mullis and colleagues at the Cetus Corporation in 1985 - may like to dust off the Journal of Molecular Biology for 1971 (vol. 56, pages 341-361) and read a paper from H. Gobind Khorana and colleagues, the last paragraph of which is reprinted here.

range of neuromuscular diseases, including the Kearns-Sayres syndrome (KSS) and chronic progressive external ophthalmoplegia (CPEO). In these cases, patients' muscle tissue contains mtDNA molecules with large-scale deletions extending over several genes ${ }^{4,5,8}$. These mutant molecules are always mixed with normal mtDNAs and represent 20-90 per cent of the total number of molecules. Curiously, there is no obvious correlation between the severity of the clinical symptoms or biochemical abnormality and either the location of the deletion or the number of deleted genes. But as transcription of mtDNA generates polycistronic transcripts corresponding to the full length of each DNA strand, a major deletion anywhere in the genome may have serious consequences for expression of genes not covered by the deletion.

In contrast to LHON and MERRF, diseases associated with deleted mtDNAs have usually not been inherited. Usually, deleted molecules within the muscle fibres of an individual have the same structure, suggesting that they arise by the clonal amplification of a single spontaneous mutational event that probably took place at some stage during fetal development. The pleiotropic nature of these diseases, thus, is likely to depend on the relative proportion of normal and deleted mtDNAs in different organs. This view is supported by recent findings ${ }^{11}$ that a deletion in mtDNA is the probable cause of a strikingly different human disease, namely Pearson's syndrome, a fatal disorder of the haemopoietic system.

Of interest for the mechanism of mutation is the fact that all deletions so far analysed in detail have their endpoints in short repeated sequences, which seem to be especially abundant in human mtDNA. One site in particular, consisting of direct repeats of 13 base pairs separated by approximately 5 kilobases, seems to be a hot spot for mutation. In a study of 30 patients with KSS or CPEO, 12 were found to have identical deletions with endpoints in a direct repeat of 13 base pairs ${ }^{5}$, whereas an identical deletion is found in a patient suffering from Pearson's syndrome $^{11}$, suggesting that this site is a hotspot for mutation. Intramolecular homologous recombination or slipped mispairing during replication, which are both plausible mechanisms for generation of large deletions in other DNAs, may be a significant source of the deletions in these diseases, but neither explanation is really satisfactory.

First, there are no reports of any DNArepair phenomena in animal mitochondria and orthodox recombination mechanisms appear to be absent ${ }^{12}$. Second, slippage mispairing, which depends on the generation of single-stranded regions in complementary strands carrying direct repeats, may not apply to animal mtDNAs, which are replicated from two origins, one on each strand, by a mechanism that is not expected to expose long stretches of complementary single-stranded DNA at any stage. But as Holt et al. have suggested ${ }^{8}$, a discontinuous synthesis mechanism may operate during periods of rapid mtDNA replication, such as during oogenesis when many of the mutations may occur.

Also of interest in terms of mutational mechanisms is the finding ${ }^{13}$ of a mother and daughter suffering from CPEO who possess mtDNA deletions of different sizes ( 2.5 and 5.0 kilobases). Thus, either the female germ line of this lineage contains two different deleted mtDNA molecules or, more likely in view of the complexity of interactions between nuclear and mitochondrial genomes during mitochondrial biogenesis, some other hereditary factor predisposes the mtDNAs of these patients to deletion.

Involvement of nuclear factors is also suggested by the results of a recent study ${ }^{6}$ of an Italian family containing several individuals affected by a late-onset mitochondrial myopathy with symptoms similar to those of KSS. Like KSS and 